\title{
On three and four point boundary value problems for second order differential inclusions
}

\author{
Mouffak Benchohra and Sotiris K. Ntouyas
}




\title{
ON THREE AND FOUR POINT BOUNDARY VALUE PROBLEMS FOR SECOND ORDER DIFFERENTIAL INCLUSIONS
}

\author{
MOUFFAK BenCHOHRA \\ Laboratoire de Mathématiques, Université de Sidi Bel Abbès \\ BP 89, 22000 Sidi Bel Abbès, Algérie \\ benchohra@yahoo.com \\ SOTIRIS K. NTOUYAS \\ Department of Mathematics, University of Ioannina \\ 45110 Ioannina, Greece \\ sntouyas@cc.uoi.gr
}

[Received March 6, 2001]

\begin{abstract}
In this paper we investigate the existence of solutions on a compact interval to a three and four-point boundary value problem for a class of second order differential inclusions. We shall rely on a fixed point theorem for contraction multivalued maps due to Covitz and Nadler.
\end{abstract}

Mathematical Subject Classification: 34A60, 34B10, 34B15

Keywords: Three and four point boundary value problems, differential inclusion, contraction, existence, fixed point

\section{Introduction}

The existence of solutions on compact intervals for three-point and multi-point boundary value problems for second order differential equations has received much attention in the last decade, we refer for instance to the papers of Boucherif and Bouguima [1], Gupta [2-5], Gupta et al [6-8], Gupta and Trofimchuk [9-10] and Marano [11]. The study of multi-point boundary value problems for second order ordinary differential equations was initiated by Il'In and Moiseev in [12-13], motivated by the work of Bitsadze and Samarskii on nonlocal elliptic boundary value problems [14-16]. The methods used are usually the topological transversality of Granas or the degree theory methods combined with Wirtinger type inequalities.

Very recently, by means of a fixed point theorem for condensing multivalued maps due to Martelli, an extension of three and four-point boundary value problems for second order differential equations to the multivalued case has been done by the authors in [17-18]. However, in these problems the right-hand side was assumed to be convex valued. Here we drop this restriction and consider problems with a nonconvex 
valued right-hand side.

In Section 3 of this paper we shall prove a theorem which ensures the existence of solutions defined on a compact real interval for the three-point boundary value problem (BVP for short) of the second order differential inclusion

$$
\begin{gathered}
y^{\prime \prime} \in F(t, y), \quad t \in J=[0,1], \\
y(0)=0, y(\eta)=y(1),
\end{gathered}
$$

where $F: J \times \mathbb{R} \longrightarrow \mathcal{P}(\mathbb{R})$ is a multivalued map, $\eta \in(0,1)$, and $\mathcal{P}(E)$ is the family of all subsets of $\mathbb{R}$. lem

Section 4 is devoted to the study of the following four-point boundary value prob-

$$
\begin{gathered}
y^{\prime \prime} \in F(t, y), \quad t \in J=[0,1], \\
y(0)=y^{\prime}(\eta), y(1)=y(\tau),
\end{gathered}
$$

where $F, \eta$ are as in the problem (1.1)-(1.2) and $\tau \in(0,1)$. The method we are going to use is to reduce the existence of solutions to problems (1.1)-(1.2) and (1.3)-(1.4) to the search for fixed points of a suitable multivalued map on the Banach space $C(J, \mathbb{R})$. In order to prove the existence of fixed points, we shall rely on a fixed point theorem for contraction multivalued maps, due to Covitz and Nadler [19].

\section{Preliminaries}

In this section, we introduce notations, definitions, and preliminary facts from multivalued analysis which are used throughout this paper.

Let $(X, d)$ be a metric space. We use the notations:

$P(X)=\{Y \in \mathcal{P}(X): Y \neq \emptyset\}, \quad P_{c l}(X)=\{Y \in P(X): Y$ closed $\}, \quad P_{b}(X)=$ $\{Y \in P(X): Y$ bounded $\}$.

Consider $H_{d}: P(X) \times P(X) \longrightarrow \mathbb{R}_{+} \cup\{\infty\}$, given by

$$
H_{d}(A, B)=\max \left\{\sup _{a \in A} d(a, B), \sup _{b \in B} d(A, b)\right\},
$$

where $d(A, b)=\inf _{a \in A} d(a, b), d(a, B)=\inf _{b \in B} d(a, b)$.

Then $\left(P_{b, c l}(X), H_{d}\right)$ is a metric space and $\left(P_{c l}(X), H_{d}\right)$ is a generalized metric space.

A multivalued map $N: J \longrightarrow P_{c l}(X)$ is said to be measurable if, for each $x \in X$, the function $Y: J \longrightarrow \mathbb{R}$, defined by

$$
Y(t)=d(x, N(t))=\inf \{|x-z|: z \in N(t)\},
$$

is measurable.

Definition 1 A multivalued operator $N: X \rightarrow P_{c l}(X)$ is called 
a) $\gamma$-Lipschitz if and only if there exists $\gamma>0$ such that

$$
H_{d}(N(x), N(y)) \leq \gamma d(x, y), \quad \text { for each } x, y \in X
$$

b) contraction if and only if it is $\gamma$-Lipschitz with $\gamma<1$.

$N$ has a fixed point if there is $x \in X$ such that $x \in N(x)$. The fixed point set of the multivalued operator $N$ will be denoted by FixN.

For more details on multivalued maps we refer to the books by Deimling [20], Gorniewicz [21] and $\mathrm{Hu}$ and Papageorgiou [22].

Our considerations are based on the following fixed point theorem for contraction multivalued operators given by Covitz and Nadler in 1970 [19] (see also Deimling, [20] Theorem 11.1).

Lemma 2 Let $(X, d)$ be a complete metric space. If $N: X \rightarrow P_{c l}(X)$ is a contraction, then $\operatorname{Fix} N \neq \emptyset$.

\section{Three-Point BVPs}

The main result of this section concerns the three-point BVP (1.1)-(1.2). Before we state and prove this result, we give the definition of a solution of the three-point BVP (1.1)-(1.2).

Definition $3 A$ function $y: J \longrightarrow \mathbb{R}$ is called a solution for the $B V P$ (1.1)-(1.2) if $y$ and its first derivative are absolutely continuous and $y^{\prime \prime}$ (which exists almost everywhere) satisfies the differential inclusion (1.1) a.e. on $J$ and the condition (1.2).

Theorem 4 Assume that:

(H1) $F: J \times \mathbb{R} \rightarrow P_{c l}(\mathbb{R})$ has the property that $F(\cdot, y): J \rightarrow P_{c l}(\mathbb{R})$ is measurable for each $y \in \mathbb{R}$.

(H2) $H_{d}(F(t, y), F(t, \bar{y})) \leq l(t)|y-\bar{y}|$, for a.e. $t \in J$ and $y, \bar{y} \in \mathbb{R}$, where $l \in$ $L^{1}(J, \mathbb{R})$.

$$
\begin{aligned}
& \text { Let } L(t)=\int_{0}^{t} l(s) d s, \text { and } \mu>1 \text {. If } \\
& \qquad \frac{1}{\mu}+\frac{L(\eta)+L(1)}{1-\eta}<1,
\end{aligned}
$$

then the BVP (1.1)-(1.2) has at least one solution on J. 
Proof. Let the Bielecki-type norm $\|\cdot\|_{B}$ on $C(J, \mathbb{R})$ be defined by

$$
\|y\|_{B}=\max _{t \in J}\left\{|y(t)| e^{-\mu L(t)}\right\} .
$$

Transform the problem into a fixed point problem. Consider the multivalued map, $N: C(J, \mathbb{R}) \longrightarrow \mathcal{P}(C(J, \mathbb{R}))$ defined by:

$$
\begin{array}{r}
N(y)=\left\{h \in C(J, \mathbb{R}): h(t)=\int_{0}^{t}(t-s) g(s) d s+\frac{t}{1-\eta} \int_{0}^{\eta}(\eta-s) g(s) d s\right. \\
\left.-\frac{t}{1-\eta} \int_{0}^{1}(1-s) g(s) d s\right\}
\end{array}
$$

where

$$
g \in S_{F, y}=\left\{g \in L^{1}(J, \mathbb{R}): g(t) \in F(t, y(t)) \text { for a.e. } t \in J\right\}
$$

Remark 5 (i) It is clear that the fixed points of $N$ are solutions to (1.1)-(1.2).

(ii) For each $y \in C(J, \mathbb{R})$ the set $S_{F, y}$ is nonempty, since by (H1), F has a measurable selection (see [23], Theorem III.6).

We shall show that $N$ satisfies the assumptions of Lemma 2. The proof will be given in two steps.

Step 1: $N(y) \in P_{c l}(C J, \mathbb{R})$ for each $y \in C(J, \mathbb{R})$.

Indeed, let $\left(y_{n}\right)_{n \geq 0} \in N(y)$ such that $y_{n} \rightarrow \tilde{y}$ in $C(J, \mathbb{R})$. Then $\tilde{y} \in C(J, \mathbb{R})$ and for each $t \in J$

$$
\begin{aligned}
y_{n}(t) \in \int_{0}^{t}(t-s) F(s, y(s)) d s+\frac{t}{1-\eta} \int_{0}^{\eta} & (\eta-s) F(s, y(s)) d s \\
& -\frac{t}{1-\eta} \int_{0}^{1}(1-s) F(s, y(s)) d s .
\end{aligned}
$$

Because the sets

$\int_{0}^{t}(t-s) F(s, y(s)) d s, \frac{t}{1-\eta} \int_{0}^{\eta}(\eta-s) F(s, y(s)) d s$ and $\frac{t}{1-\eta} \int_{0}^{1}(1-s) F(s, y(s)) d s$ are closed for each $t \in J$, then for each $t \in J$

$$
\begin{aligned}
y_{n}(t) \rightarrow \tilde{y}(t) \in \int_{0}^{t}(t-s) F(s, y(s)) d s+ & \frac{t}{1-\eta} \int_{0}^{\eta}(\eta-s) F(s, y(s)) d s \\
& -\frac{t}{1-\eta} \int_{0}^{1}(1-s) F(s, y(s)) d s .
\end{aligned}
$$

Therefore $\tilde{y} \in N(y)$. 
Step 2: $H_{d}\left(N\left(y_{1}\right), N\left(y_{2}\right)\right) \leq \gamma\left|y_{1}-y_{2}\right|$ for each $y_{1}, y_{2} \in C(J, \mathbb{R})$ (where $\left.\gamma<1\right)$.

Let $y_{1}, y_{2} \in C(J, \mathbb{R})$ and $h_{1} \in N\left(y_{1}\right)$. Then there exists $g_{1}(t) \in F\left(t, y_{1}(t)\right)$ such that, for each $t \in J$,

$$
h_{1}(t)=\int_{0}^{t}(t-s) g_{1}(s) d s+\frac{t}{1-\eta} \int_{0}^{\eta}(\eta-s) g_{1}(s) d s-\frac{t}{1-\eta} \int_{0}^{1}(1-s) g_{1}(s) d s
$$

From (H2) it follows that

$$
H_{d}\left(F\left(t, y_{1}(t)\right), F\left(t, y_{2}(t)\right)\right) \leq l(t)\left|y_{1}(t)-y_{2}(t)\right|
$$

Hence, there is $w \in F\left(t, y_{2}(t)\right)$, such that

$$
\left|g_{1}(t)-w\right| \leq l(t)\left|y_{1}(t)-y_{2}(t)\right|, \quad t \in J
$$

Consider $U: J \rightarrow \mathcal{P}(\mathbb{R})$, given by

$$
U(t)=\left\{w \in \mathbb{R}:\left|g_{1}(t)-w\right| \leq l(t)\left|y_{1}(t)-y_{2}(t)\right|\right\}
$$

Since the multivalued operator $V(t)=U(t) \cap F\left(t, y_{2}(t)\right)$ is measurable (see Proposition III.4 in [23] there exists $g_{2}(t)$ a measurable selection for $V$. So, $g_{2}(t) \in F\left(t, y_{2}(t)\right)$ and

$$
\left|g_{1}(t)-g_{2}(t)\right| \leq l(t)\left|y_{1}(t)-y_{2}(t)\right|, \text { for each } t \in J
$$

Let us define for each $t \in J$

$$
h_{2}(t)=\int_{0}^{t}(t-s) g_{2}(s) d s+\frac{t}{1-\eta} \int_{0}^{\eta}(\eta-s) g_{2}(s) d s-\frac{t}{1-\eta} \int_{0}^{1}(1-s) g_{2}(s) d s
$$

Then we have

$$
\begin{aligned}
\left|h_{1}(t)-h_{2}(t)\right| \leq & \int_{0}^{t}(t-s)\left|g_{1}(s)-g_{2}()\right| d s+\frac{t}{1-\eta} \int_{0}^{\eta}(\eta-s)\left|g_{1}(s)-g_{2}(s)\right| d s \\
& +\frac{t}{1-\eta} \int_{0}^{1}(1-s)\left|g_{1}(s)-g_{2}(s)\right| d s \\
\leq & \int_{0}^{t} l(s)\left|y_{1}(s)-y_{2}(s)\right| d s+\frac{1}{1-\eta} \int_{0}^{\eta}(\eta-s) l(s)\left|y_{1}(s)-y_{2}(s)\right| d s \\
& +\frac{1}{1-\eta} \int_{0}^{1}(1-s) l(s)\left|y_{1}(s)-y_{2}(s)\right| d s \leq
\end{aligned}
$$




$$
\begin{aligned}
\leq & \int_{0}^{t} l(s) e^{-\mu L(s)} e^{\mu L(s)}\left|y_{1}(s)-y_{2}(s)\right| d s \\
& +\frac{1}{1-\eta} L(\eta) e^{\mu L(t)}\left\|y_{1}-y_{2}\right\|_{B} \\
& +\frac{1}{1-\eta} L(1) e^{\mu L(t)}\left\|y_{1}-y_{2}\right\|_{B} \\
\leq \quad & \left\|y_{1}-y_{2}\right\|_{B} \int_{0}^{t} l(s) e^{\mu L(s)} d s+\frac{1}{1-\eta} L(\eta) e^{\mu L(t)}\left\|y_{1}-y_{2}\right\|_{B} \\
& +\frac{1}{1-\eta} L(1) e^{\mu L(t)}\left\|y_{1}-y_{2}\right\|_{B} \\
=\quad & \left\|y_{1}-y_{2}\right\|_{B} \frac{1}{\mu} \int_{0}^{t}\left(e^{\mu L(s)}\right)^{\prime} d s+\frac{1}{1-\eta} L(\eta) e^{\mu L(t)}\left\|y_{1}-y_{2}\right\|_{B} \\
& +\frac{1}{1-\eta} L(1) e^{\mu L(t)}\left\|y_{1}-y_{2}\right\|_{B} \\
\leq \quad & \frac{\left\|y_{1}-y_{2}\right\|_{B}}{\mu} e^{\mu L(t)}+\frac{1}{1-\eta} L(\eta) e^{\mu L(t)}\left\|y_{1}-y_{2}\right\|_{B} \\
& +\frac{1}{1-\eta} L(1) e^{\mu L(t)}\left\|y_{1}-y_{2}\right\|_{B} .
\end{aligned}
$$

Then

$$
\left\|h_{1}-h_{2}\right\|_{B} \leq\left[\frac{1}{\mu}+\frac{L(\eta)+L(1)}{1-\eta}\right]\left\|y_{1}-y_{2}\right\|_{B} .
$$

By the analogous relation, obtained by interchanging the roles of $y_{1}$ and $y_{2}$, it follows that

$$
H_{d}\left(N\left(y_{1}\right), N\left(y_{2}\right)\right) \leq\left[\frac{1}{\mu}+\frac{L(\eta)+L(1)}{1-\eta}\right]\left\|y_{1}-y_{2}\right\|_{B} .
$$

So, $N$ is a contraction and thus, by Lemma 2 , it has a fixed point $y$, which is solution to (1.1)-(1.2).

\section{Four-Point BVPs}

The main result of this section concerns the four-point BVP (1.3)-(1.4). Before we state and prove this result, we give the definition of a solution of the four-point BVP (1.3)-(1.4).

Definition 6 A function $y: J \longrightarrow \mathbb{R}$ is called a solution for the BVP (1.3)-(1.4) if $y$ and its first derivative are absolutely continuous and $y^{\prime \prime}$ (which exists almost everywhere) satisfies the differential inclusion (1.3) a.e. on $J$ and the conditions (1.4).

Theorem 7 Assume that (H1) and (H2) are satisfied. If

$$
\frac{1}{\mu}+L(\eta)+2 \frac{L(\tau)+L(1)}{1-\eta}<1
$$


then the BVP (1.3)-(1.4) has at least one solution on $J$.

Proof. Let $\|\cdot\|_{B}$ the Bielecki-type norm on $C(J, \mathbb{R})$ defined by

$$
\|y\|_{B}=\max _{t \in J}\left\{|y(t)| e^{-\mu L(t)}\right\} .
$$

Transform the problem into a fixed point problem. Consider the multivalued map, $N_{1}: C(J, \mathbb{R}) \rightarrow \mathcal{P}(C(J, \mathbb{R}))$ defined by:

$$
\begin{aligned}
N_{1}(y)= & \left\{h \in C(J, \mathbb{R}): h(t)=\int_{0}^{t}(t-s) g(s) d s+\int_{0}^{\eta} g(s) d s\right. \\
& \left.+\frac{1+t}{1-\tau}\left[\int_{0}^{\tau}(\tau-s) g(s) d s-\int_{0}^{1}(1-s) g(s) d s\right]\right\}
\end{aligned}
$$

where

$$
g \in S_{F, y}=\left\{g \in L^{1}(J, \mathbb{R}): g(t) \in F(t, y(t)) \text { for a.e. in } J\right\} .
$$

We can easily show that $N_{1}$ has closed values and it is a contraction multivalued map. We omit the details.

\section{Concluding Remarks}

Let $a_{i} \in \mathbb{R}$, with all of the $a_{i}^{\prime} s$ having the same sign, $\xi_{i} \in(0,1), i=1,2, \ldots, m-2$, $0<\xi_{1}<\xi_{2}<\ldots<\xi_{m-2}<1$. Consider the following m-point boundary value problem for second order differential inclusions

$$
\begin{gathered}
y^{\prime \prime}(t) \in F(t, y(t)), \quad t \in J \\
y(0)=0, \quad y(1)=\sum_{i=1}^{m-2} a_{i} y\left(\xi_{i}\right)
\end{gathered}
$$

It is well known (see [12] for example) that if a function $y \in C^{1}$ satisfies the boundary condition (5.2) and all of the $a_{i}, i=1,2, \ldots, m-2$ have the same sign, then there exists $\eta \in\left[\xi_{1}, \xi_{m-2}\right]$, depending on $y \in C^{1}(J, \mathbb{R})$ such that

$$
y(1)=\alpha y(\eta)
$$

with $\alpha=\sum_{i=1}^{m-2} a_{i}$. Accordingly, the problem of the existence of a solution for the BVP (5.1)-(5.2) can be studied via the three-point BVP

$$
\begin{gathered}
y^{\prime \prime}(t)=F(t, y(t)), \quad t \in J, \\
y(0)=0, \quad y(1)=\alpha y(\eta),
\end{gathered}
$$

where $\eta \in(0,1)$ is given. We omit the details, since the proof follows the steps of the proof of Theorem 4, with obvious modifications. 
It is obvious that the above method can also be applied to other types of m-point BVPs. For example for the BVPs

$$
\begin{gathered}
y^{\prime \prime}(t) \in F(t, y(t)), \quad t \in J, \\
y^{\prime}(0)=0, y(1)=\sum_{i=1}^{m-2} a_{i} y\left(\xi_{i}\right),
\end{gathered}
$$

or

$$
\begin{gathered}
y^{\prime \prime}(t) \in F(t, y(t)), \quad t \in J \\
y(0)=0, y^{\prime}(1)=\sum_{i=1}^{m-2} a_{i} y^{\prime}\left(\xi_{i}\right)
\end{gathered}
$$

which can be reduced to the following three point BVPs:

$$
\begin{gathered}
y^{\prime \prime}(t) \in F(t, y(t)), \quad t \in J, \\
y^{\prime}(0)=0, \quad y(1)=\alpha y(\eta)
\end{gathered}
$$

and

$$
\begin{gathered}
y^{\prime \prime}(t)=F(t, y(t)), \quad t \in J, \\
y(0)=0, \quad y^{\prime}(1)=\alpha y^{\prime}(\eta)
\end{gathered}
$$

respectively.

\section{REFERENCES}

[1] Boucherif, A. and Bouguima, S. M.: Nonlinear second order ordinary differential equations with nonlocal boundary conditions, Commu. Appl. Nonl. Anal., $\mathbf{5}(2),(1998), 73-85$.

[2] Gupta, C. P.: Solvability of a three-point boundary value problem for a second order ordinary differential equation, J. Math. Anal. Appl., 168, (1992), 540-551.

[3] Gupta, C. P.: A note on a second order three-point boundary value problem, J. Math. Anal. Appl., 186, (1994), 277-281.

[4] Gupta, C. P.: A second order m-point boundary value problem at resonance, Nonlinear Anal., 24, (1994), 1483-1489.

[5] Gupta, C. P.: Solvability of a multi-point boundary value problem at resonance, Results Math., 28, (1995), 270-276.

[6] Gupta, C. P., Ntouyas, S. K. and Tsamatos, P. Ch.: On an m-point boundary value problem for second order ordinary differential equations, Nonlinear Anal. 23 (1994), 1427-1436.

[7] Gupta, C. P., Ntouyas, S. K. and Tsamatos, P. Ch.: Existence results for m-point boundary value problems, Differential Equations Dynam. Systems, 2, (1994), 289-298. 
[8] Gupta, C. P., Ntouyas, S. K. and Tsamatos, P. Ch.: Solvability of an mpoint boundary value problem for second order ordinary differential equations, J. Math. Anal. Appl., 189, (1995), 575-584.

[9] Gupta, C. P. and Trofimchuk, S. I.: A sharper condition for the solvability of a three-point second order boundary value problem, J. Math. Anal. Appl., 285, (1997), 586-597.

[10] Gupta, C. P. and Trofimchuk, S. I.: A Wirtinger type inequality and a threepoint boundary value problem, Dynam. Systems Appl., 8, (1999), 127-132.

[11] Marano, S. A.: A remark on a second order three-point boundary value problem, J. Math. Anal. Appl., 183, (1994), 518-522.

[12] Il'in, V. A. and Moiseev, E. I.: Nonlocal boundary value problem of the first kind for a Sturm-Liouville operator in its differential and finite difference aspects, Differential Equations, 23(7), (1987), 803-810.

[13] IL'In, V. A. and Moiseev, E. I.: Nonlocal boundary value problem of the second kind for a Sturm-Liouville operator, Differential Equations, 23(8), (1987), 979987.

[14] Bitsadze, A. V.: On the theory of nonlocal boundary value problem, Soviet. Math. Dokl., 30(1), (1984), 8-10.

[15] Bitsadze, A. V.: On a class of conditionally solvable nonlocal boundary value problems for harmonic functions, Soviet. Math. Dokl., 31(1), (1985), 91-94.

[16] Bitsadze, A. V. and SAmarskit, A. A.: On some simple generalizations of linear elliptic boundary value problem, Soviet. Math. Dokl., 10(2), (1969), 398400 .

[17] Benchohra, M. and Ntouyas, S. K.: A note on a three-point boundary value problem for second order differential inclusions, Mathematical Notes, Miskolc, 2, (2001), 39-47.

[18] Benchohra, M. and Ntouyas, S. K.: Multi-point boundary value problems for second order differential inclusions, Math. Vesnik, to appear.

[19] Covitz, H. and Nadler S. B. JR.: Multivalued contraction mappings in generalized metric spaces, Israel J. Math., 8, (1970), 5-11.

[20] Deimling, K.: Multivalued Differential Equations, Walter de Gruyter, BerlinNew York, 1992.

[21] Gorniewicz, L.: Topological Fixed Point Theory of Multivalued Mappings, Mathematics and its Applications, 495, Kluwer Academic Publishers, Dordrecht, 1999.

[22] Hu Sh. and Papageorgiou, N.: Handbook of Multivalued Analysis, Volume I: Theory, Kluwer Academic Publishers, Dordrecht, 1997.

[23] Castaing, C. and Valadier, M.: Convex Analysis and Measurable Multifunctions, Lecture Notes in Mathematics, Vol. 580, Springer-Verlag, BerlinHeidelberg-New York, 1977. 